\section{PTH-062 PREVALENCE AND PATHOGENESIS OF ANAEMIA IN INFLAMMATORY BOWEL DISEASE: A CROSS SECTIONAL STUDY IN A LARGE TERTIARY CENTRE}

doi:10.1136/gut.2011.239301.463

S Tanwar, ${ }^{1 *}$ G Lipman, ${ }^{1}$ J Parkes, ${ }^{2}$ S McCartney' ${ }^{1}$ University College Hospital, London, UK; ${ }^{2}$ University of Southampton, Southampton, UK

Introduction Both anaemia and iron deficiency are being increasingly recognised in patients with inflammatory bowel disease (IBD) not only as manifestations of active disease but as factors influencing patient quality of life in their own right. ${ }^{1}$ As a result, guidelines have been formulated to facilitate the management of patients in these settings. ${ }^{2}$ In this study the authors have investigated the prevalence and the treatment requirements of both anaemia and iron deficiency in outpatients with IBD.

Methods In a cross-sectional study, all outpatients with IBD at our centre were identified. After excluding for pregnancy, recent surgery and blood dyscrasias, 543 outpatients with IBD were identified (265 with ulcerative colitis, 268 with Crohn's disease, 10 with indeterminate colitis). In addition to both gender and age, haemoglobin, iron status and iron therapy were also collected. Disease activity was confirmed by endoscopic and/or radiological assessment. C reactive protein (CRP) was also collected as a presumed surrogate for disease activity.

Results Of the 543 patients with IBD, 91 patients (16.8\%) were anaemic of which 45 patients $(49.5 \%)$ had biochemical evidence of iron deficiency. 38 patients $(14.3 \%)$ with ulcerative colitis compared with 52 patients $(19.5 \%)$ with Crohn's disease were anaemic $(p=0.102)$. Anaemia was associated with the presence of iron deficiency $(p<0.001)$ and active disease $(p=0.005)$ but not with gender. Similarly, iron deficiency was also associated with active disease $(p<0.001)$ but not gender or the type of IBD. CRP was significantly different in patients with and without both anaemia and iron deficiency $(p<0.001)$. In patients with active disease, both haemoglobin $(p=0.010)$ and CRP $(p<0.001)$ were significantly different to those with inactive disease but not MCV or ferritin. Of the 38 patients who were on oral iron, $11(29 \%)$ had a suboptimal response and required intravenous iron. 6 out of 12 patients being administered intravenous iron required erythropoetin.

Conclusion Anaemia is a common finding in outpatients with IBD with frequency of up to $20 \%$ of which almost half had evidence of iron deficiency. In contrast to oral iron therapy, intravenous iron and erythropoietin therapy were required for only $2 \%$ and $1 \%$ of patients respectively. Anaemia was associated with an older age, active disease and iron deficiency. Both 
anaemia, iron deficiency and CRP appear to act as surrogate markers for active disease.

Competing interests None.

Keywords anaemia, Crohn's disease, disease activity,

inflammatory bowel disease, iron deficiency, ulcerative colitis.

\section{REFERENCES}

1. Wells CW, Lewis S, Barton JR, et al. Effects of changes in hemoglobin level on quality of life and cognitive function in inflammatory bowel disease patients. Inflamm Bowel Dis 2006;12:123-30.

2. Gache $C$, Berstad A, Befrits R, et al. Guidelines on the diagnosis and management of iron deficiency and anemia in inflammatory bowel diseases. Inflamm Bowel Dis 2007;13:1545-53. 